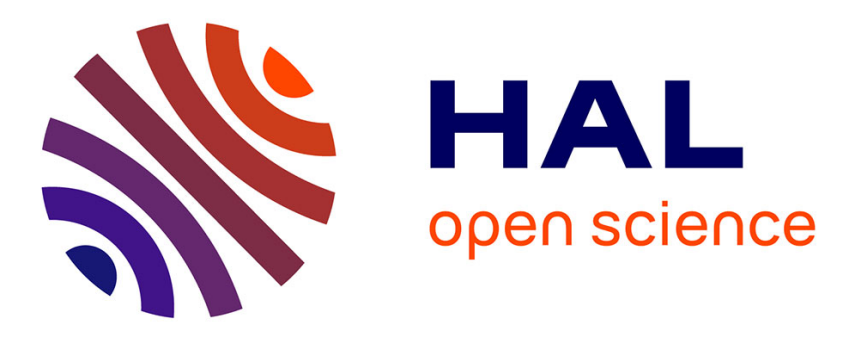

\title{
Dynamo threshold detection in the von Kármán sodium experiment
}

\author{
Sophie Miralles, Nicolas Bonnefoy, Mickaël Bourgoin, Philippe Odier, \\ Jean-François Pinton, Nicolas Plihon, Gautier Verhille, Jean Boisson, François \\ Daviaud, Bérengère Dubrulle
}

\section{To cite this version:}

Sophie Miralles, Nicolas Bonnefoy, Mickaël Bourgoin, Philippe Odier, Jean-François Pinton, et al.. Dynamo threshold detection in the von Kármán sodium experiment. Physical Review E: Statistical, Nonlinear, and Soft Matter Physics, 2013, 88, pp.013002. 10.1103/PhysRevE.88.013002 . hal00941503

\section{HAL Id: hal-00941503 \\ https://hal.science/hal-00941503}

Submitted on 13 Apr 2015

HAL is a multi-disciplinary open access archive for the deposit and dissemination of scientific research documents, whether they are published or not. The documents may come from teaching and research institutions in France or abroad, or from public or private research centers.
L'archive ouverte pluridisciplinaire HAL, est destinée au dépôt et à la diffusion de documents scientifiques de niveau recherche, publiés ou non, émanant des établissements d'enseignement et de recherche français ou étrangers, des laboratoires publics ou privés. 


\title{
Dynamo threshold detection in the von Kármán sodium experiment
}

\author{
Sophie Miralles, Nicolas Bonnefoy, ${ }^{*}$ Mickael Bourgoin ${ }^{\dagger}$ Philippe Odier, Jean-François Pinton, \\ Nicolas Plihon, ${ }^{\ddagger}$ and Gautier Verhille ${ }^{\S}$ \\ Laboratoire de Physique, École Normale Supérieure de Lyon, CNRS \& Université de Lyon, 46 allée d'Italie, 69364 Lyon Cedex 07, France
}

Jean Boisson," François Daviaud, and Bérengère Dubrulle

SPHYNX, Service de Physique de l'État Condensé, CNRS \& CEA Saclay, 91191 Gif-sur-Yvette Cedex, France

(Received 7 March 2013; published 8 July 2013)

\begin{abstract}
Predicting dynamo self-generation in liquid metal experiments has been an ongoing question for many years. In contrast to simple dynamical systems for which reliable techniques have been developed, the ability to predict the dynamo capacity of a flow and the estimate of the corresponding critical value of the magnetic Reynolds number (the control parameter of the instability) has been elusive, partly due to the high level of turbulent fluctuations of flows in such experiments (with kinetic Reynolds numbers in excess of $10^{6}$ ). We address these issues here, using the von Kármán sodium experiment and studying its response to an externally applied magnetic field. We first show that a dynamo threshold can be estimated from analysis related to critical slowing down and susceptibility divergence, in configurations for which dynamo action is indeed observed. These approaches are then applied to flow configurations that have failed to self-generate magnetic fields within operational limits, and we quantify the dynamo capacity of these configurations.
\end{abstract}

DOI: 10.1103/PhysRevE.88.013002

PACS number(s): 47.65.-d, 47.27.-i, 82.40.Bj

\section{INTRODUCTION}

Estimating the proximity to a possible drastic change of behavior in a complex system (a bifurcation) is a generic and open question. Determination of precursors of instabilities (either transient or steady state) is essential in many applied fields, and also gives valuable information for the understanding of the physical mechanisms driving the instability. For some specific hydrodynamics instabilities, the critical values for the control parameter at which a supercritical instability develops may be computed from the dynamical constitutive or model equations describing the system; see for instance [1]. In other situations, for example when fluctuations or boundary conditions (BCs) play an (unknown) leading role, the computation of critical values for control parameters may become intractable.

Possible methods for instability detection have been suggested using the close analogy between the nonlinear bifurcations of model systems and phase transitions. This is the case of critical slowing down and susceptibility divergence which have been thoroughly checked for some continuous instabilities. A normalized control parameter is usually introduced as $\epsilon=\left(A-A_{c}\right) / A_{c}$, where $A$ is the value of the control parameter and $A_{c}$ the critical value at which the instability develops. One approach - usually restricted to positive values

\footnotetext{
*Also at CEA/CE Cadarache, DTN/STPA/LIET, 13108 St. Paul lez Durance Cedex, France.

${ }^{\dagger}$ Also at Laboratoire des Ecoulements Géophysiques et Industriels, CNRS, and Université Joseph Fourier, BP 53, 38041 Grenoble Cedex 9, France.

${ }^{\ddagger}$ nicolas.plihon@ens-lyon.fr

${ }^{\S}$ Also at Institut de Recherche sur les Phénomènes Hors Equilibre, CNRS, and Aix-Marseille Université, 49 rue F. Joliot Curie, B.P. 146, 13384 Marseille Cedex 13, France.

"Also at Unité de Mécanique (funded by the chaire AREVA), ENSTA-ParisTech, 828 Boulevard des Maréchaux, 91762 Palaiseau Cedex, France.
}

of $\epsilon$-is based on the scaling of the amplitudes of physical parameters, such as the growth of the amplitude of the unstable mode, as a function of $\epsilon$. Another concerns the transient dynamics, as for instance the critical slowing down, which has been observed for variations of the control parameter from (or to) subcritical values $(\epsilon<0)$ to (or from) supercritical values $(\epsilon>0)$. They have been checked for some supercritical bifurcations such as Rayleigh-Bénard convection [2-4] and in Taylor-Couette flows [5-7]. In these studies, one usually needs to cross the instability threshold and the methods may not be suitable to determine critical values from behavior below threshold. Allain and coworkers [8] overcame this limitation by the analysis of the temporal decay of forced thermal variations in Rayleigh-Bénard convection below threshold. They showed that the decay-time decreases as $\epsilon^{-1}$ and gave correct estimates for the critical value of thermal convection. The authors also pointed out that the decay-time evolution strongly depends on the spatial patterns of forced thermal variations, and was highest when the spatial wavelength of the forcing matched that of the most unstable pattern. Critical slowing down near threshold has also been observed in the transition to turbulence in planar Poiseuille flows (following the spatial decay/growth rates of forced fluctuations) [9] and more recently in pipe flows [10]. The estimate of the critical control parameter was shown to be in good agreement with the real critical value at which the instability was observed.

In the context of magnetohydrodynamics (MHD), and more specifically in the context of the dynamo instability, the precise determination of the critical value of the magnetic Reynolds number above which magnetic field grows spontaneously is an important issue. The study of growth/decay rates has long been used in kinematic dynamo studies where the induction equation is used to compute growth rates of the magnetic fields modes, from a prescribed velocity field (as the solution of the Navier-Stokes equation with vanishing Lorentz force). In other words, the induction equation is analyzed as an eigenvalue problem, for which the largest eigenvalue is numerically 
computed, the critical value being the value of the control parameter for which the most positive eigenvalue is equal to zero (i.e., when critical slowing down is observed). This is for instance the case of the swirling flows considered in [11], which are related to the von Kármán flows, of the optimization of the von Kármán sodium experiment [12], and of the models of the von Kármán sodium dynamo [13-15], in contrast to the case of [16] which considered a two-way coupling between the velocity and the magnetic fields.

In dynamo experiments, currently restricted to liquid metals, the accessible magnetic Reynolds numbers are mostly limited by the available power. The fine tuning of dynamo flows has been an important part of dynamo experiments, the estimate of the critical magnetic Reynolds number being a critical issue. In their initial study, Gailitis and coworkers [17] studied the evolution of advected harmonic magnetic field perturbations as a function of magnetic Reynolds number, below threshold, in a cylindrical helical flow mimicking the Ponomarenko flow [18]. They observed that the inverse of the amplitude linearly decreases with the magnetic Reynolds number, with strong variations as a function of the frequency of the magnetic perturbations. Unfortunately this experimental setup failed to generate a dynamo and the method could not be checked regarding the estimate of a dynamo threshold. In their later experiment, the Riga group successfully showed that both magnetic susceptibility divergence and critical slowing down could be used to estimate the dynamo threshold [19]. It should be emphasized here that the initial stage of the magnetic field growth of the Riga dynamo was shown to be correctly described by laminar theory [20]. Evolution of the decay time from low-amplitude, large-scale applied magnetic field was also analyzed in secondary pumps of the fast breeder reactor Superphenix [21], as well as in the unconstrained and more turbulent Maryland experiment [22]. These measurements showed variations of the relaxation time as a function of the magnetic Reynolds number but were however restricted to situations where dynamo instability was not reached. It is the purpose of this paper to analyze in detail the reliability of these methods in determining the threshold in the turbulent von Kármán sodium (VKS) experiment, in which, by changing boundary conditions and driving schemes, dynamo self-generation has either been reached or has remained outside of the accessible experimental parameters range.

In Sec. II, the von Kármán sodium experimental dynamo setup is described. In Sec. III, we focus on a dynamo configuration, for which the threshold is shown to be accurately estimated from analysis of induced magnetic field dynamics and amplitude. In Sec. IV, we use the observations of Sec. III in a detailed analysis of the influence of boundary conditions on dynamo generation. Discussion and conclusions are given in the last section.

\section{EXPERIMENTAL SETUP}

The von Kármán sodium experiment is schematically displayed in Fig. 1. Liquid sodium flows are created by the counterrotation of two coaxial impellers within a copper cylinder of radius $R_{o}=289 \mathrm{~mm}$ and length $524 \mathrm{~mm}$. Each impeller is composed of a thick disk (of radius $R_{\mathrm{imp}}=$ $154.5 \mathrm{~mm}$ and thickness $18 \mathrm{~mm}$ ) fitted with eight blades with

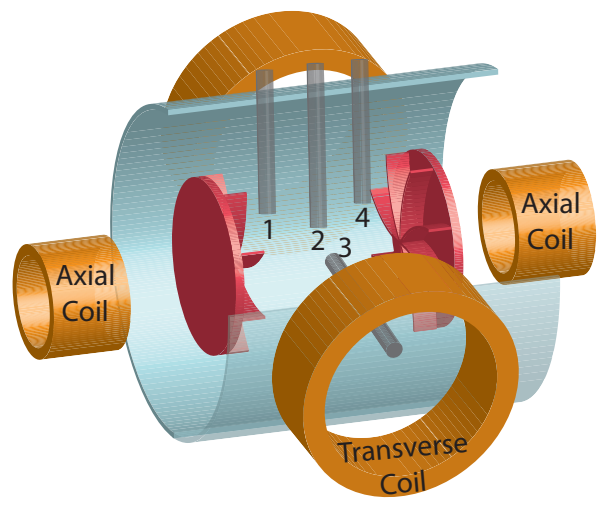

FIG. 1. (Color online) Schematic of the experimental setup, in flow configuration 2 (see text for details). Probe shafts represent the location of the magnetic field sensor arrays.

height $41.2 \mathrm{~mm}$. They are driven up to typically $25 \mathrm{~Hz}$ by motors with $300 \mathrm{~kW}$ available power, and oil circulation in the outer copper cylinder regulates the sodium temperature around $120^{\circ} \mathrm{C}$.

An integral magnetic Reynolds number is defined as $\mathrm{Rm}=\mu_{0} \sigma 2 \pi F R_{\mathrm{imp}} R_{o} .{ }^{1}$ The temperature dependence of the electrical conductivity of sodium $\sigma$ is taken into account in the computation of Rm. Since the magnetic Prandtl number of sodium is of the order of $10^{-5}$, the integral kinetic Reynolds number is in excess of $10^{6}$; i.e., the MHD flows considered are highly turbulent, with a rate of velocity fluctuations (rms to mean) of order unity.

It has been previously shown [23] that dynamo action was observed above a critical magnetic Reynolds number $\mathrm{Rm}_{\mathrm{c}}$ around 40. In exact counterrotation of the two impellers, the dynamo magnetic field is statistically stationary, with a rate of fluctuations of order unity. When the impellers are driven asymmetrically, dynamical regimes such as magnetic field reversals or oscillations have also been observed [23]. Self-generation has only been observed when the flow is driven by the rotation of soft-iron impellers [24]. In the remaining of the paper, unless otherwise stated, we restrict our study to the case of exact counterrotation, first considering cases for which the dynamo threshold has been reached (in Sec. III). Section IV then focuses on the influence of modified electromagnetic and hydrodynamic boundary conditions (obtained by inserting appendices in the flow or changing impellers and/or vessel materials) in cases for which self-generation has not been observed. We emphasize that the term "electromagnetic boundary conditions" refers here both to static and rotating mechanical parts. As pointed out in Sec. IV, static or rotating ferromagnetic parts do not have the same influence on the magnetic response of similar flows. However, since an exact understanding of the influence of moving immersed conductive/ferromagnetic material in

\footnotetext{
${ }^{1}$ In earlier publications of the VKS collaboration, the integral magnetic Reynolds number had been defined as $\mathrm{Rm}=\mathcal{K} \mu_{0} \sigma 2 \pi R_{o}^{2} F$ where $\mathcal{K}=0.6$ is a coefficient that measures the efficiency of the impellers, resulting in slightly different values for the critical magnetic Reynolds numbers.
} 
conductive fluids is not available, we thus encompass both cases as the effect of "boundary conditions."

Magnetic fields are recorded using four arrays of ten 3-axis Hall effect sensors inserted in radial shafts, as shown in Fig. 1. Two arrays are inserted in the midplane of the vessel, within long probe shafts (labeled 2 and 3 in Fig. 1), the deepest probe being $63 \mathrm{~mm}$ away from the cylinder's axis, and neighboring probes being separated by $28 \mathrm{~mm}$. Two arrays are inserted closer to the impellers (109 $\mathrm{mm}$ away from the midplane of the vessel), within shorter probe shafts (labeled 1 and 4 in Fig. 1), the deepest probe being $103 \mathrm{~mm}$ away from the cylinder's axis. These magnetic field sensors are recorded using National Instruments PXI6259 16 bit digitizers at a rate of $2000 \mathrm{~Hz}$. The accuracy of the measured magnetic fields is $\pm 0.1 \mathrm{G}$. Two pairs of external coils allow for the application of controlled magnetic fields in the experimental setup in order to study the magnetic response of the flow (the applied magnetic field amplitudes ranging from $1.5 \mathrm{G}$ to $5 \mathrm{G}$ ). One pair of coils is aligned with the cylinder's axis. Depending on the relative sign of the current in the axial coils, a magnetic field having the geometry of an axial dipole can be imposed from the Helmholtz axial setup (current in the same direction in both coils) or a magnetic field having the geometry of an axial quadrupole can be imposed from the anti-Helmholtz axial setup (current in opposite directions in the two coils). The other pair of coils is perpendicular to the cylinder's axis; the Helmholtz transverse setup creates a magnetic field having the structure of a transverse dipole. If $\theta$ is the angular position around the rotation axis, we define the azimuthal mode number $m$ such that the magnetic field dependance is proportional to $e^{i m \theta}$. With this notation, the axial coils provide an $m=0$ field (either dipole or quadrupole), while the transverse coils create an $m=1$ field.

\section{BEHAVIOR FOR DYNAMO RUNS}

We consider here cases where the flow is driven using impellers made of an iron disk with iron blades, for which dynamo action has been observed. We first describe the results for a configuration with curved blades, where dynamo action is observed above a critical value $\mathrm{Rm}_{\mathrm{c}} \simeq 44$ [25,26] (run labeled $R$ in Sec. IV). We show that induction measurements allow for a reliable estimate of this critical value, using two methods. The first method relies on the determination of relaxation dynamics from applied magnetic pulses and the observation of critical slowing down, while the second method analyzes global magnetic induction measurements and divergence of susceptibility. The results are presented as a function of the normalized control parameter $\epsilon=\left(\mathrm{Rm}-\mathrm{Rm}_{\mathrm{c}}\right) / \mathrm{Rm}_{\mathrm{c}}$. The two methods are described in detail in the next two subsections, before being applied in the last subsection to dynamo configurations with straight blades and to nondynamo runs in next section.

\section{A. Decay time measurements}

\section{Description of the method}

We study here the decay of an externally applied magnetic field pulse, and estimate its eventual variation with $\mathrm{Rm}$ in the vicinity of threshold. The external field is applied using the two Helmholtz axial coils (refer to Fig. 1) which generate an axial dipole field, i.e., having a large projection onto the dynamo magnetic field.

Figure 2(a) shows a typical time evolution of the axial component of the magnetic field $\mathbf{B}(t)$ at one location for one single realization of the relaxation from one applied pulse, below threshold (namely $\epsilon=-0.13$ ), together with the relaxation of the current applied in the axial coils. One observes a relaxation of the magnetic field from an ON state which is the response to a large scale applied magnetic field at $t<0$ (the ON state is around $50 \mathrm{G}$ ) to an OFF state without externally applied magnetic field (the OFF state being around $10 \mathrm{G}$ ). Due to the high level of flow fluctuations, the details of this relaxation vary very much from one pulse to the next.

We first extract a mean behavior, averaging the relaxation recorded by all sensors in the flow. In this manner, contributions from all locations in the flow are taken into account, in a procedure that is appropriate for a global bifurcation in which a large-scale magnetic dipole is generated. We thus define and study the global magnetic energy

$$
E_{B}(t)=\frac{1}{40} \sum_{N=1}^{4} \sum_{i=1}^{10}\left|\mathbf{B}_{i, N}(t)-\left\langle\mathbf{B}_{i, N}^{\mathrm{OFF}}\right\rangle\right|^{2},
$$

where $|\mathbf{B}|$ is the norm of the magnetic field $\mathbf{B}, \mathbf{B}_{i, N}(t)$ is the instantaneous magnetic field and $\mathbf{B}_{i, N}^{\mathrm{OFF}}$ the relaxed magnetic field without externally applied field at location $i$ within probe array $N$, and $\langle\cdot\rangle$ stands for time averaged. Figure 2(b) shows an average of the relaxation dynamics of $E_{B}(t)$ over 30 realizations, at $\epsilon=-0.13$ (full blue line). The energy level is normalized to its average value at $t<0$ when the coils current is ON. As expected, the turbulent fluctuations are averaged out - they are incoherent in space and from one pulse to the next. The energy decay is close to an exponential behavior (as observed in the inset) and we extract a characteristic decay time $\tau$ from a least-squares fit of the functional form $\exp \left[-\left(t-t_{0}\right) / \tau\right]$. An example of fit is shown as the dashed red curve in Fig. 2(b). It is also possible to quantify the fluctuations of decay times about the mean behavior, out of 400 realizations (obtained in run $U$; see Sec. IV). For every single realization a decay time is estimated by a best fit of $E_{B}(t)$ to an exponential decay. The distributions of values are shown in Fig. 2(c), for $\epsilon=-0.41$ and $\epsilon=-0.11$ (the distributions are normalized to the value of the decay time with no flow). As one nears threshold the mean decay time and the standard deviation increase (a 33\% increase for the mean value and a twofold increase for the standard deviation from $\epsilon=-0.41$ to $\epsilon=-0.11)$. Although the probability distribution functions (pdf) are close to a Gaussian [full red curves in Fig. 2(c)], they are not completely symmetric. The skewness of the actual distribution is positive and increases as the distance to threshold decreases-it is 0.5 at $\epsilon=-0.41$, and 0.9 at $\epsilon=-0.11$.

\section{Threshold determination}

In the following, we focus on the evolution of mean decay times when the rotation rate of the impellers (and hence Rm and $\epsilon$ ) increases. We first consider the response to an applied external field, having a similar geometry to the dynamo mode, as produced by the axial Helmholtz coils shown in Fig. 1. 

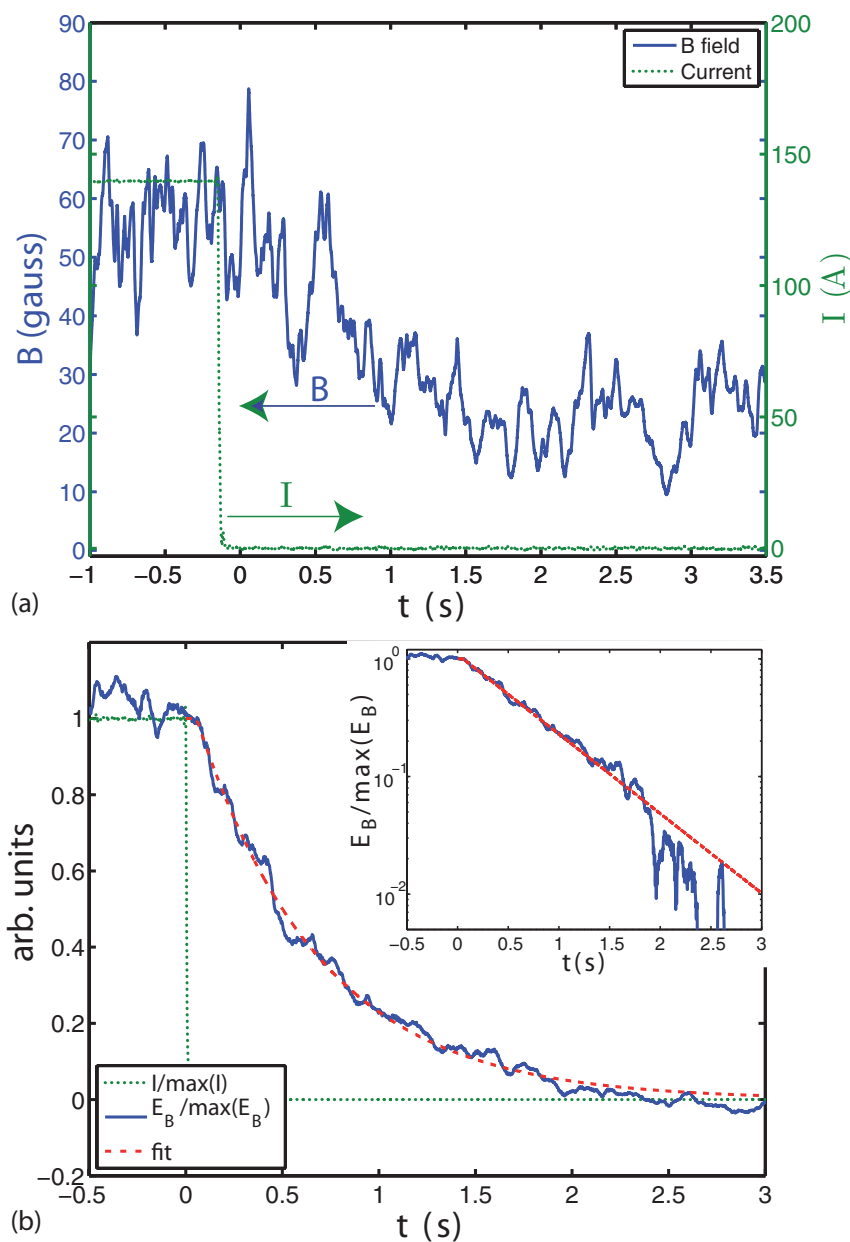

(b)

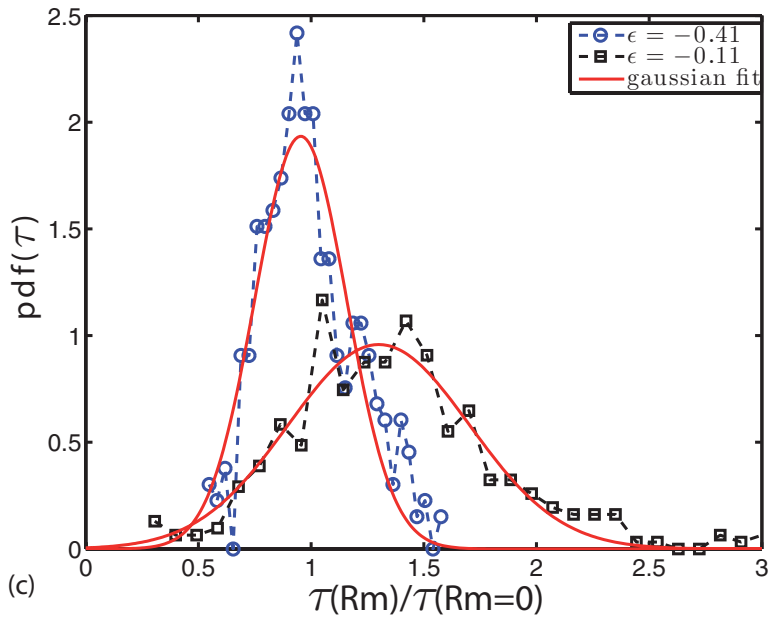

FIG. 2. (Color online) Relaxation dynamics at $\epsilon=-0.13$ for a dynamo run (run $R$ ) and applied $m=0$ dipole magnetic field: (a) time traces for a single realization: magnetic field at one location [full (blue) curve] and current in axial coils [dotted (green) curve], and (b) normalized realization-averaged global magnetic energy and coil current (30 realizations)- the inset shows the curves in lin-log scale; see text for details. (c) Individual probability distribution functions (dashed curves) of the decay time $\tau$ (normalized to the decay time with no sodium motion) and associated Gaussian distributions [full (red) curve]—dynamo run $U$.

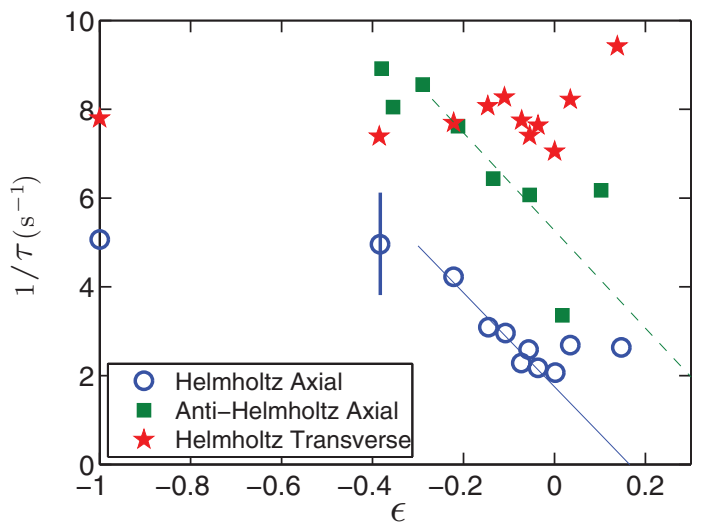

FIG. 3. (Color online) Evolution of relaxation decay time from an applied Helmholtz axial magnetic field (open blue circles), having an $m=0$ dipolar structure, an applied anti-Helmholtz axial magnetic field (green squares), having an $m=0$ quadrupolar structure, and an applied Helmholtz transverse magnetic field (red stars), having an $m=1$ dipolar structure, as a function of $\epsilon$. The lines are the linear extrapolation of the $1 / \tau$ evolution in the range $-0.3<\epsilon<0$.

For each value of $\epsilon$, the relaxation dynamics from pulsed applied fields has been averaged over 30 independent realizations. Results are shown in Fig. 3, open (blue) circles, the error bar being estimated as the standard deviation over the independent realizations. In the absence of motion, one measures $\tau=0.2 \mathrm{~s}$, which corresponds to the ohmic diffusion of magnetic fields over a length $L_{d}=\sqrt{2 \tau / \mu_{0} \sigma} \approx 0.2 \mathrm{~m} \sim$ $R_{o}$. As one nears threshold, our main observations are as follows:

(i) The decay time increases clearly with values that start to be noticeably different from simple ohmic decay at about $20 \%$ below threshold.

(ii) $1 / \tau$ does not reach (or nears) zero, but saturates to a final value starting from the dynamo threshold and onwards.

(iii) A linear extrapolation of the $1 / \tau$ evolution crosses zero for $\epsilon \sim 0.17$, leading thus to an overestimate of the threshold. The extrapolation is based on the linear best fit of $1 / \tau$ for $-0.3<\epsilon<0$, shown as a full line in Fig. 3 .

Some of these features can be understood by a simple model. Let us consider the following imperfect supercritical bifurcation:

$$
\dot{x}=\alpha+\epsilon x-x^{3},
$$

where $x$ stands for the time-averaged magnetic field.

The stationary solutions $x_{0}$, computed according to Cardan's formulas, are displayed as a full line in Fig. 4 (using $\alpha=0.005$ ). The normalized amplitude of the magnetic field during OFF phases is also displayed as open symbols; the dynamical system governed by Eq. (1) thus correctly captures the dynamo bifurcation. We emphasize here that the imperfect nature of the dynamo bifurcation has already been observed in the Karlsruhe dynamo [27]. At first order, the linear relaxation dynamics of the system governed by (1) corresponds to an exponential decay with characteristic time $\tau_{l}^{-1}=-\epsilon+3 x_{0}^{2}$, shown in the inset of Fig. 4 as a dash-dotted line. This evolution shows that no divergence of the relaxation time can be observed near threshold for an imperfect supercritical bifurcation. 


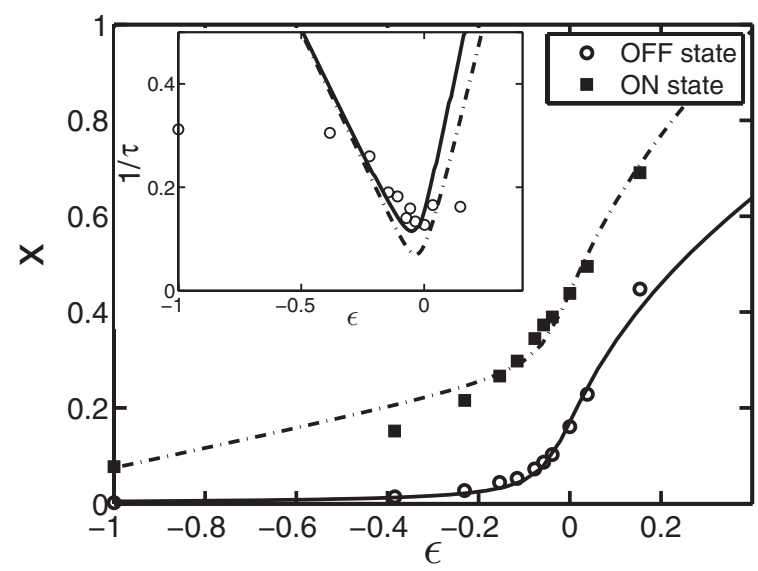

FIG. 4. Experimental (points) and imperfect supercritical model (lines) for the OFF and ON states (with an axial applied dipolar magnetic field). Inset: Evolution of the decay time for the imperfect supercritical model, with $\alpha=0.005$.

In Fig. 4, the experimental response to the applied Helmholtz axial field - ON states_- has also been plotted (filled squares). The observed behavior can be accurately accounted for as resulting from 3 contributions: (i) the OFF states (i.e., the growing dynamo), (ii) a constant applied magnetic field, (iii) a magnetic field induced from the applied field (and linearly scaling with $\mathrm{Rm}$ ). This simple linear model is displayed as a dashed line in Fig. 4 and correctly describes the experimental data.

Since in the experiment the relaxation is not observed from a vanishingly small perturbation $\left(\left\langle B_{\mathrm{ON}}\right\rangle /\left\langle B_{\mathrm{OFF}}\right\rangle\right.$ is of order unity), one solves numerically the model equation (1) from an initial condition corresponding to the ON state. The relaxation is then fitted by an exponential decay, and the results are displayed as a full line in the inset of Fig. 4. The effect of finite initial conditions adds up to the fact that a complete divergence of the relaxation time, down to the smallest distance to threshold, cannot be observed.

We note here, as a partial conclusion, that relaxation from an applied magnetic field having a similar geometry to the dynamo mode displays critical slowing down below dynamo threshold. This critical slowing down is observed when the forcing reaches at least $0.75 \mathrm{Rm}_{\mathrm{c}}$, and no divergence is observed at threshold. The observed critical slowing down can be used to detect the dynamo threshold with a simple criterion. This criterion, based on linear extrapolation, leads to an overestimate of the threshold by $17 \%$.

\section{Influence of the applied field geometry}

We now investigate how the relaxation dynamics is affected when the geometry of the applied magnetic field differs from the geometry of the dynamo mode. As mentioned in the introduction, the projection of the applied perturbation on the unstable mode was shown to be an important parameter in many situations $[8,17,22]$.

We first describe measurements in the anti-Helmholtz axial setup, for which the externally applied magnetic field is generated from the axial coils running currents in opposite directions, thus producing a geometry resembling an axial
( $m=0)$ quadrupole. The variations of the decay times in this case are displayed as (green) squares in Fig. 3. Decay times times evolve similarly to the case of the Helmholtz axial setup. The linear fit of the $1 / \tau$ evolution in the range $-0.3<\epsilon<0$ (dashed green line in Fig. 3) crosses zero at $\epsilon=0.48$. We emphasize here that the geometry of the induced magnetic field during the ON phase is quadrupolar and that this geometry was checked to be preserved during the relaxation (i.e., the magnetic response of the system preserves the symmetries of the applied magnetic field).

The situation is quite different for the Helmholtz transverse setup, for which the applied magnetic field has the geometry of a transverse, $m=1$, dipole. In this situation, no significant change in the decay time is observed either below or above threshold; cf. red stars in Fig. 3. We recall here that the most unstable magnetic mode computed from kinematic simulations taking into account the time-average axisymmetric velocity field [12] is an $m=1$ dipole (satisfying Cowling's theorem), while the observed experimental mode is an $m=0$ dipole. This difference in relaxation dynamics between axial and transverse dipoles has previously been observed in a similar experiment [22] in which dynamo action was not reached.

To conclude, the evolution of the relaxation dynamics shows that the projection of the applied magnetic perturbation on the unstable dynamo mode is, as in other situations $[8,17,22]$, a crucial parameter, and that critical slowing down has been observed only for axially axisymmetric applied magnetic field. No divergence of the relaxation time is observed at threshold, a reminiscent fact of the imperfect nature of the dynamo bifurcation. The linear extrapolation of the $1 / \tau$ evolution leads to an overestimate of the dynamo threshold, by $17 \%$ in the Helmholtz axial configuration and $48 \%$ in the anti-Helmholtz axial configuration.

\section{B. Induction measurements}

We now investigate another method for the estimate of the dynamo threshold based on the strength of the induced field in response to an externally applied one. We focus on the Helmholtz axial and anti-Helmholtz axial cases, and consider the inverse of a susceptibility $\chi$ defined as:

$$
1 / \chi=\frac{1}{40} \sum_{N=1}^{4} \sum_{i=1}^{10} \frac{\left|\mathbf{B}_{i, N}^{\mathrm{app}}\right|}{\left\langle\left|B_{i, N}^{x}\right|\right\rangle+\left\langle\left|B_{i, N}^{y}\right|\right\rangle+\left\langle\left|B_{i, N}^{z}\right|\right\rangle},
$$

where $\left|\mathbf{B}_{i, N}^{\text {app }}\right|$ is the norm of the applied magnetic field and $B_{i, N}^{x, y, z}$ are the components of the magnetic response $\mathbf{B}_{i, N}$ to the applied magnetic field at location $i$ within probe array $N$.

Several choices may be considered for $\mathbf{B}_{i, N}$. The simplest one uses the raw value of the magnetic response, i.e., the induction recorded by the Hall arrays when the applied field is turned ON, such that $\mathbf{B}_{i, N}=\mathbf{B}_{i, N}^{\mathrm{ON}}$. This leads to a definition of $1 / \chi_{1}$, which is displayed in the top graph of Fig. 5 as a function of $\epsilon$ for Helmholtz axial and anti-Helmholtz axial applied magnetic fields. Both curves show a linear decrease below threshold, which can be used as an estimate for the dynamo threshold. The $1 / \chi_{1}$ evolutions have thus been linearly fitted in the range $-0.4<\epsilon<0$. The extrapolation of the linear fits leads to an overestimate of the threshold, namely $\epsilon \sim 0.19$ and 0.37 for the Helmholtz axial and anti-Helmholtz axial 

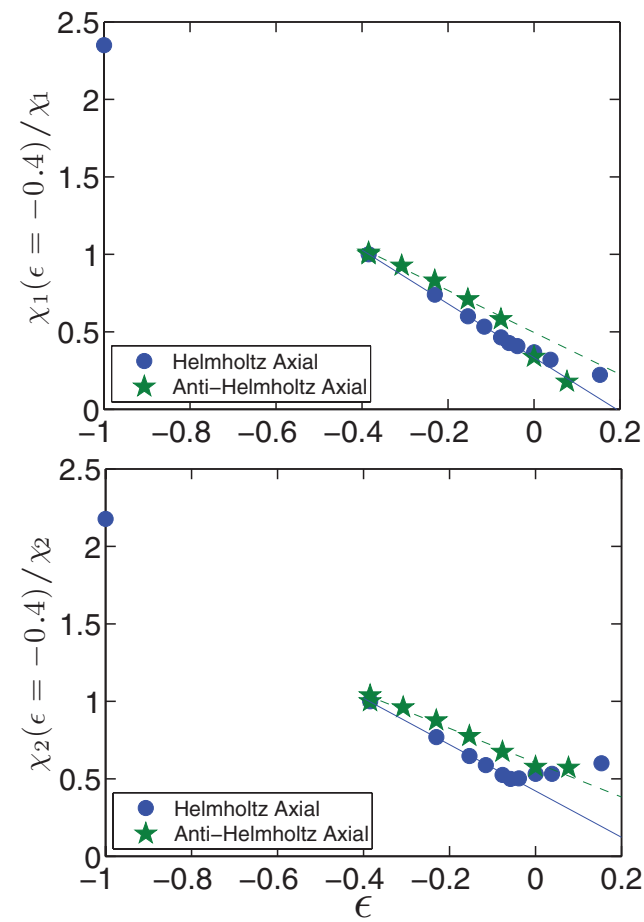

FIG. 5. (Color online) $1 / \chi$ as a function of $\epsilon$ for the dynamo run, Helmholtz axial, and anti-Helmholtz axial setups. Curves have been normalized to their values at $\epsilon=-0.4$; see text for detailed definitions of $\chi_{1}$ and $\chi_{2}$. Linear fits (in the range $-0.4<\epsilon<0$ ) shown as full and dashed lines.

setup, respectively. Similarly to the critical slowing down, no divergence of the susceptibility is observed at threshold.

Since close to self-generation, the dynamo contribution to the amplitudes of $\mathbf{B}_{i, N}^{\mathrm{ON}}$ quickly increases with $\mathrm{Rm}$, it is instructive to introduce a susceptibility $\chi_{2}$ defined with $\mathbf{B}_{i, N}=\mathbf{B}_{i, N}^{\mathrm{ON}}-\mathbf{B}_{i, N}^{\mathrm{OFF}}$. This definition takes into account the geometry of the applied magnetic field in a better way since it has been checked that the induced magnetic field $\left(\mathbf{B}_{i, N}^{\mathrm{ON}}-\mathbf{B}_{i, N}^{\mathrm{OFF}}\right)$ has the same symmetries as the applied magnetic field. The evolution of $1 / \chi_{2}$ is shown in the bottom graph of Fig. 5. The behavior is similar to the one of $1 / \chi_{1}$, except that saturation is observed for positive values of $\epsilon$, which was not observed in the evolution of $\chi_{1}$ due to the dominant contribution of the dynamo field above threshold. The linear fit of the $1 / \chi_{2}$ evolution (computed for $-0.4<\epsilon<0$ ) crosses zero at $\epsilon=0.28$ and $\epsilon=0.54$ for the Helmholtz axial and anti-Helmholtz axial cases, respectively.

These results thus show that a dynamo threshold may also be estimated from induction measurements and the evolution of a magnetic susceptibility. In a way similar to the analysis of relaxation dynamics, thresholds estimated in the Helmholtz axial configuration are lower than those estimated in the antiHelmholtz axial configurations.

\section{Application to dynamo runs with straight blades}

One distinctive feature of the VKS dynamo is that the dynamo instability has only been observed when the flow is driven by impellers entirely made of soft iron (i.e., disk and blades). The value of threshold does depend on the exact nature of the flow-for instance the direction of rotation for impellers with curved blades or the presence of appendices in the vessel-but the use of soft-iron impellers has proved essential, so far. In this subsection, we discuss the application of the two methods previously introduced to dynamo runs with straight blades (namely runs $U$ and $V$; refer to Sec. IV for details).

Dynamo instability has indeed been observed when the flow is driven by soft-iron impellers fitted with straight blades, with a higher critical magnetic Reynolds number $(\sim 60$ for run $V$ ) than with curved blades ( $\sim 44$ for run $R$ with impellers rotating in the unscooping direction and $\sim 68$ with impellers rotating in the scooping direction). As a consequence, the operational power limits are reached for lower values of $\epsilon=\left(\mathrm{Rm}-\mathrm{Rm}_{\mathrm{c}}\right) / \mathrm{Rm}_{\mathrm{c}}$ with straight blades than with curved blades $[\max (\epsilon) \sim 0.4$ for run $V$ and and $\max (\epsilon) \sim 0.8$ for run $R$ at exact counterrotation]. A second observation is that no dynamical regimes have been observed for runs $U$ and $V$ when driving the flow asymmetrically. This may be due to the power limits, since dynamical regimes were observed for run $R$ above $\epsilon \sim 0.55$, as secondary instabilities from stationary dynamo states; based on this argument, observation of dynamical regimes in run $V$ would require $35 \%$ more mechanical power than presently available.

Despite those discrepancies, the two methods introduced above gave similar results for the three dynamo runs $R, U$, and $V$ :

(i) Critical slowing down and magnetic susceptibility increase were observed both for the Helmholtz axial setup and for the anti-Helmholtz axial setup.

(ii) The linearly extrapolated threshold for the antiHelmholtz axial setup is slightly larger than the linearly extrapolated threshold for the Helmholtz axial setup.

(iii) No critical slowing down has been observed for the Helmholtz transverse setup.

(iv) During the relaxation, the magnetic response of the system preserves the symmetries of the applied magnetic field.

The linear extrapolations of the $1 / \tau$ and the $1 / \chi_{2}$ evolutions lead to an overestimate of the dynamo threshold, by $17-35 \%$ in the Helmholtz axial configuration and 32-66 \% in the antiHelmholtz axial configuration depending on the dynamo run. This may reflect the threshold difference between two different dynamo modes, namely an axial $(m=0)$ dipole and an axial $(m=0)$ quadrupole [28] or the difference in coupling between symmetric or antisymmetric modes generated in the vicinity of each impeller $[25,30]$. Given those results, we postulate that dynamical regimes were not observed in runs $U$ and $V$ due to operational limits; i.e., the maximum $\epsilon$ values are too small, or equivalently, the maximum $\mathrm{Rm}$ values are too close to the critical value.

As a final remark it should be emphasized that for both methods, the dynamo threshold is overestimated by around 15-30\%. This overestimate might be problematic in terms of accessible operating parameters since, in the turbulent flows of interest, the power budget scales as $\mathrm{Rm}^{3}$. A $20 \%$ overestimate in $\mathrm{Rm}$ thus corresponds to a $73 \%$ overestimate in power requirements. 


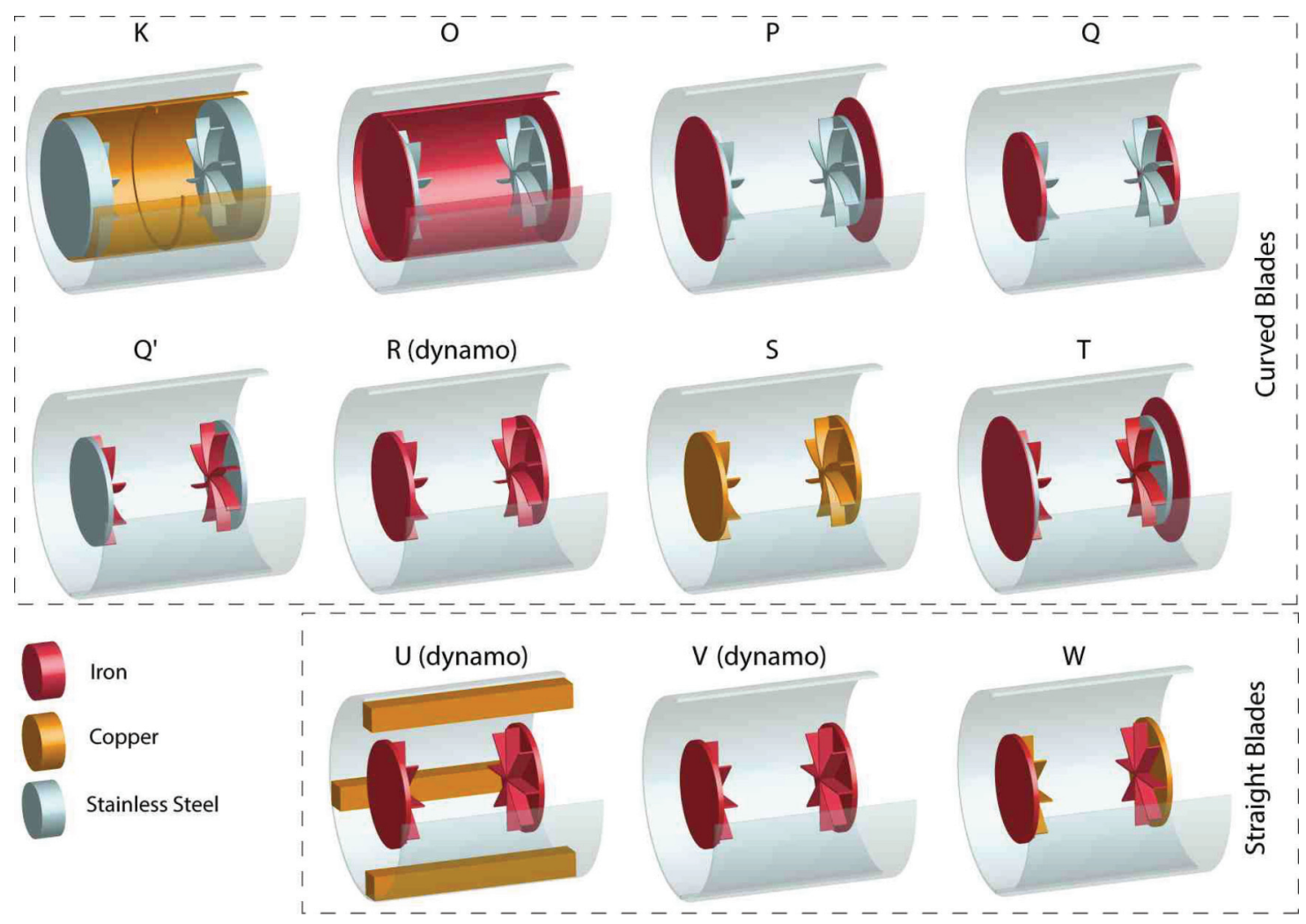

FIG. 6. (Color online) Schematic of the eleven studied VKS configurations. Gray color stands for stainless steel (except for the outer copper cylinder, which is displayed in gray for clarity of the figure), yellow color for copper, and red for soft iron. Static soft-iron screens behind the impellers can clearly be seen for runs $O, P$, and $T$.

\section{INFLUENCE OF BOUNDARY CONDITIONS ON DYNAMO GENERATION}

As emphasized above, dynamo instability has only been observed in the VKS experiment when the flow was driven by impellers (both disk and blades) entirely made of soft iron. A number of VKS runs (with various configurations) have thus been dedicated to the study of the influence of electromagnetic boundary conditions, in regards to the observation of selfgeneration and possible dynamical regimes [25]. As already discussed in Sec. II, here we use "electromagnetic boundary conditions" in a rather loose manner, since it refers to modification of the electrical conductivity and/or the magnetic permeability of both static and rotating parts in contact with liquid sodium. In this section, using the methods introduced in the previous section, we investigate which of these runs are closest to dynamo threshold. The experimental configurations are first introduced in Sec. IV A. The difference between static and rotating high magnetic permeability material on dynamo capability is then analyzed in Sec. IV B using the methods introduced in the previous section. Finally, in Sec. IV C, the difference between high magnetic permeability and high electrical conductivity material is presented.

\section{A. Description of experimental configurations}

The experimental configurations investigated are displayed in Fig. 6, summarized in Table I, for 11 VKS runs (labeled with capital letters). These configurations differ by (i) the geometry and material of the impellers, and (ii) the details of the outer vessel enclosing the flow.

Three kinds of impellers have been used. For most of the runs, the eight blades are curved, and the impellers rotate in the unscooping direction. Run $K$ was operated with a thicker disk body, extending up to the lateral side of the outer copper cylinder - the blades being curved. For runs $U, V$, and $W$, the impellers were fitted with straight blades. Between different runs, the materials of the cylinder, the disk of the impellers, and the blades can be varied independently and are summarized in the second column of Table I. The flow volume itself extends laterally to the external stainless steel vessel $\left(R_{c}=R_{o}=289 \mathrm{~mm}\right)$, except for runs $K$ and run $O$ for which an inner cylinder (coaxial with the outer cylinder) of radius $R_{i}=206 \mathrm{~mm}$ confines the flow and sets $R_{c}=R_{i}$; in these configurations, a layer of sodium at rest is present for $R_{i}<r<R_{o}$. The inner cylinder is $5 \mathrm{~mm}$ thick and is made of copper for run $K$ and of soft iron for run $O$. For run $U$, four longitudinal copper baffles (square cross section $60 \times 40 \mathrm{~mm}^{2}$, length $250 \mathrm{~mm}$ ) have been attached to the outer cylinder. These modifications are summarized in the third column of Table I. The last type of configuration change relates to the $50 \mathrm{~mm}$ lid layer between the back of the impellers and the lateral sides of the vessel. For most of the configurations, liquid sodium in this region is set into motion by the impeller rotation. For run $K$, the use of a thicker impeller dramatically reduced the thickness of the lid layer down to $5 \mathrm{~mm}$. For runs $O, P$, and $T$, a soft-iron screen disk (of radius $206 \mathrm{~mm}$, thickness $5 \mathrm{~mm}$ ) 
TABLE I. Description of investigated configurations. SS stands for stainless steel, Cu for copper, and Fe for soft iron. When no experimental data are available, the cell mentions "no data." "None" is mentioned in cases when no decrease of either $1 / \tau$ or $1 / \chi$ is observed. See text for details on flows. The configurations marked with an asterisk (*) are the ones discussed in Figs. 7 and 8 . The maximum reachable Rm is $\sim 80$ except for runs $K, O$, and $W$ for which it is $\sim 70$.

\begin{tabular}{|c|c|c|c|c|c|c|c|}
\hline Run & $\begin{array}{c}\text { Impellers } \\
\text { (disk/blades) }\end{array}$ & Cylinder BC & Lateral BC & Flow & $\begin{array}{l}\text { Dynamo } \\
\text { threshold }\end{array}$ & $\begin{array}{l}\text { Threshold estim. } \\
\text { (decay time) }\end{array}$ & $\begin{array}{l}\text { Threshold estim. } \\
\text { (induction) }\end{array}$ \\
\hline$K$ & $\begin{array}{c}\mathrm{SS} / \mathrm{SS} \\
\text { thick impellers }\end{array}$ & $\mathrm{Cu}$ inner cyl. & thick impellers & $1^{\prime \prime}$ & & $62\left(\right.$ transverse $\left.^{\mathrm{a}}\right)$ & no data \\
\hline$O^{*}$ & SS/SS & Fe inner cyl. & Fe screen & $1^{\prime}$ & & $\sim 81$ & none \\
\hline$P^{*}$ & $\mathrm{SS} / \mathrm{SS}$ & & Fe screen & $2^{\prime}$ & & none & none \\
\hline$Q^{*}$ & $\mathrm{Fe} / \mathrm{SS}$ & & & 2 & & none & $\sim 190$ \\
\hline$Q^{\prime}$ & $\mathrm{SS} / \mathrm{Fe}$ & & & 2 & & $\sim 350$ & $\sim 125$ \\
\hline$R^{*}$ & $\mathrm{Fe} / \mathrm{Fe}$ & & & 2 & $\mathrm{Rm}_{\mathrm{c}}=44^{\mathrm{b}}$ & $\sim 51$ & $\sim 56$ \\
\hline$S$ & $\mathrm{Cu} / \mathrm{Cu}$ & & & 2 & & no data & no data \\
\hline$T$ & $\mathrm{SS} / \mathrm{Fe}$ & & Fe Screen & $2^{\prime}$ & & $\sim 250$ & $\sim 205$ \\
\hline$U$ & $\mathrm{Fe} / \mathrm{Fe}$ straight & $\mathrm{Cu}$ baffles & & $3^{\prime \prime \prime}$ & $\mathrm{Rm}_{\mathrm{c}}=70$ & $\sim 85$ & $\sim 100$ \\
\hline$V$ & $\mathrm{Fe} / \mathrm{Fe}$ straight & & & $2^{\prime \prime \prime}$ & $\mathrm{Rm}_{\mathrm{c}}=60$ & $\sim 71$ & $\sim 93$ \\
\hline$W$ & $\mathrm{Fe} / \mathrm{Cu}$ straight & & & $2^{\prime \prime \prime}$ & & none & $\sim 120^{\mathrm{c}}$ \\
\hline$W$ & $\mathrm{Cu} / \mathrm{Fe}$ straight & & & $2^{\prime \prime \prime}$ & & none & $\sim 97^{\mathrm{c}}$ \\
\hline
\end{tabular}

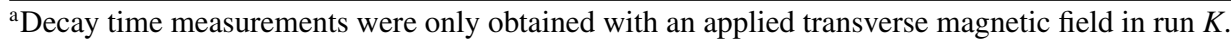

${ }^{\mathrm{b}}$ When curved-bladed impellers are rotated in the scooping direction [(-) direction in [23,25]], $\mathrm{Rm}_{\mathrm{c}}=68$.

${ }^{c}$ Estimated from induction measurement in the closest probe shaft to the referred-to impeller; see text for details.

is set at rest $5 \mathrm{~mm}$ behind the impellers. These configurations are specified in the fourth column of Table I.

In all cases, the sodium flows created by the counterrotation of the impellers have a time-averaged structure of the "s2t2" type according to the Dudley James classification [11]. However, the details of the flow structure and of the fluctuations depend on the boundary conditions and vary from one configuration to the other [29]. These flow configurations are summarized in the fifth column of Table I, where "flow 1 " denotes the flow created in presence of an inner cylinder, "flow 2" denotes the flow created without the inner cylinder, and "flow 3" denotes the flow created with the four longitudinal baffles. $X^{\prime}$ denotes the presence of the soft-iron screens behind the impellers, $X^{\prime \prime}$ denotes the use of thick impellers, and $X^{\prime \prime \prime}$ the use of straight blades.

All runs, except run $W$, were symmetric configurations; i.e., both impellers were identical. This is not the case for run $W$ where one impeller was made of a copper disk fitted with eight straight soft-iron blades (on cylinder's end; hereafter denoted $W-\mathrm{CuFe}$ ) and the other impeller was made of a soft-iron disk fitted with eight straight copper blades (on cylinder's end; hereafter denoted $W$ - $\mathrm{FeCu}$ ).

An integral magnetic Reynolds number is defined as $\mathrm{Rm}=\mu_{0} \sigma 2 \pi F R_{\mathrm{imp}} R_{c}$ where $R_{c}$ is the radius of the sodium flow (i.e., $R_{c}=R_{o}$, except for runs $K$ and $O$, where $R_{c}=R_{i}$ ).

Among the eleven configurations described in Table I, only three of are prone to dynamo action, namely run $R$ (which was studied in detail in Sec. III), run $U$, and run $V$ (impellers with straight blades; see Sec. III), for which both the disks and blades are machined from soft iron. The respective dynamo thresholds are presented in the sixth column of Table I.

For the sake of clarity, only a few of these runs will be detailed, namely runs $O, P, Q$, which are indicated in Table I. These runs will also systematically be compared to dynamo run $R$.

\section{B. Static versus rotating soft iron}

In this subsection, we will focus on runs where soft-iron material has been used either for the impellers or as static appendices, together with stainless steel. Let us first focus on pulse-decay measurements performed in the Helmholtz axial case (for which critical slowing down was observed in dynamo runs). The evolution of decay times with $\mathrm{Rm}$ is displayed for four runs (namely $O, P, Q$, and $R$, with curved blades) in Fig. 7. Two types of behavior are observed:

(i) In runs $(P, Q), 1 / \tau$ increases slightly with $\mathrm{Rm}$, giving no indication towards self-generation (reported as "none" in the seventh column of Table I). A similar increase has also been observed for runs $Q^{\prime}$ and $T$. Its magnitude (about 30\%) is consistent with an enhanced turbulent magnetic diffusivity [32-34].

(ii) In run $O, 1 / \tau$ decreases with $\mathrm{Rm}$. Critical slowing down is observed and a threshold can be estimated from linear extrapolation (similarly to the procedure applied to run $R$ in the previous section); its value is displayed in the seventh column of Table I. For run $O$, the linear extrapolation

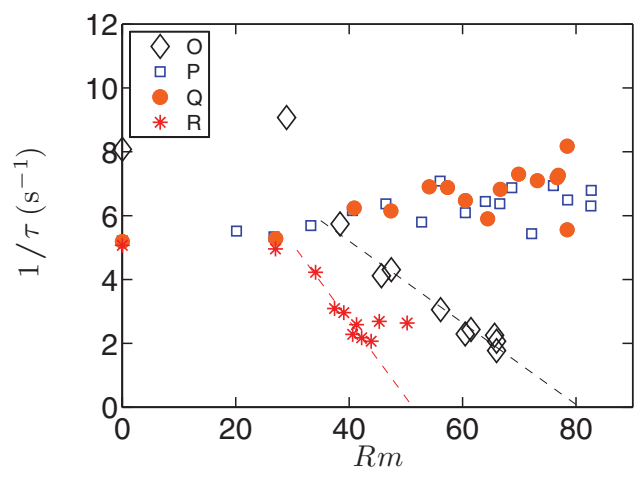

FIG. 7. (Color online) Decay time evolution with $\mathrm{Rm}$ for runs $O$, $P, Q, R$ and applied Helmholtz axial magnetic field. 


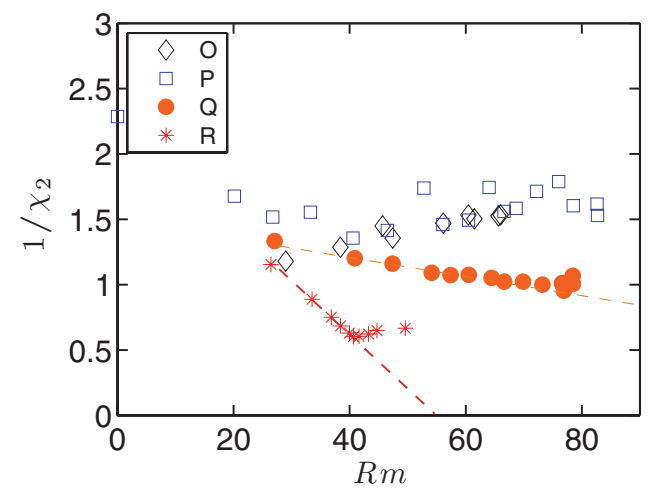

FIG. 8. (Color online) Evolution of $1 / \chi_{2}$ with Rm for runs $O, P$, $Q, R$ and applied Helmholtz axial magnetic field.

crosses zero around $\mathrm{Rm} \sim 81$, which may mean a threshold as low as $\mathrm{Rm} \sim 69$, if one corrects for a $\sim 20 \%$ overestimate (based on the observations made earlier on run $R$ ). Among the nondynamo investigated configurations, run $O$ was the only one that showed a strong decrease of $1 / \tau$ when applying an axial dipole. $^{2}$

Pulse decay measurements in the Helmholtz transverse case (applied $m=1$ dipole) have also been performed for several runs (not shown in Fig. 7), but no evolution with Rm has been measured except for run $K$; see next subsection.

Let us now investigate the magnetic susceptibility evolution with $\mathrm{Rm}$ for the same runs. The evolutions of $1 / \chi_{2}$ (cf. Sec. III B) as a function of $\mathrm{Rm}$ are displayed in Fig. 8 for runs $O, P, Q$. Similarly to decay times, two typical behaviors are observed, namely an increase or a decrease of $1 / \chi_{2}$. An increase of $1 / \chi_{2}$ with $\mathrm{Rm}$ corresponds to a case without dynamo instability; in this case the mention "none" is again displayed in the eighth column of Table I. When $1 / \chi_{2}$ decreases with $\mathrm{Rm}$, a divergence of susceptibility is possible and a threshold can be estimated from linear interpolation of the $1 / \chi_{2}$ decrease to zero. The estimated threshold is reported in the eighth column of Table I. The evolution of $1 / \chi_{2}$ for run $O$ does not point to an instability threshold (contrary to $1 / \tau)$. The evolution of $1 / \chi_{2}$ increases for run $P($ as $1 / \tau)$ and decreases for run $Q$ (contrary to $1 / \tau$ ).

These discrepancies motivate a closer analysis of the induction processes for runs $P$ and $Q$. We will focus on the azimuthal field component, as the $\omega$ effect, particularly in the vicinity of the impellers [30,31], plays a major role in the VKS dynamo generation. In the midplane of the experimental setup, the azimuthal component increases with $\mathrm{Rm}$ for all investigated runs (not shown). ${ }^{3}$ However, nearer the rotating impellers (i.e., in probe shafts labeled 1 and 4 in Fig. 1), the behaviors differ, as shown in Fig. 9. In contrast to run $P$, in run $Q$, the radial profile of the normalized induced azimuthal component increases with $\mathrm{Rm}$, a feature common to all dynamo runs, as well as with runs with rotating soft-iron

\footnotetext{
${ }^{2}$ Note that decay time were not investigated during run $S$.

${ }^{3}$ Note that the axial induced component behaves similarly for all investigated configurations, and does not depend on details of the boundary conditions.
}
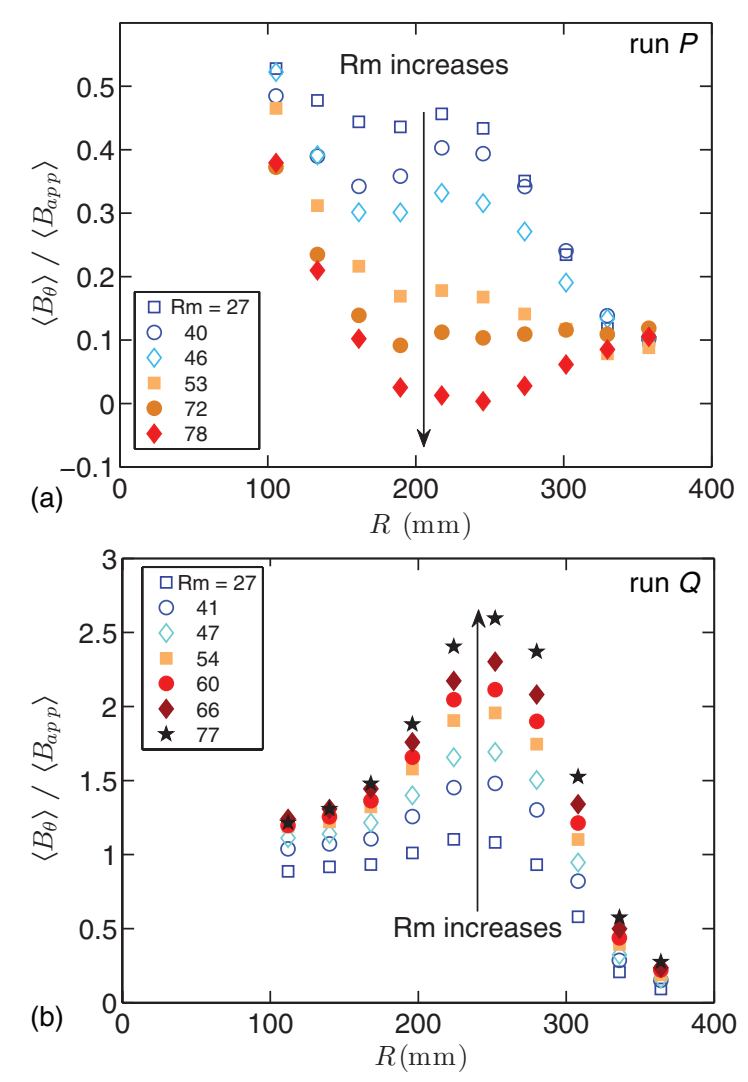

FIG. 9. (Color online) Normalized induced azimuthal magnetic field radial profile in probe shafts closer to one impeller as function of $\mathrm{Rm}$ for (a) run $P$, (b) run $Q$.

parts in the impellers, such as runs $Q^{\prime}$ and $T$. We propose that this feature is related to the decrease of $1 / \chi_{2}$ for run $Q$ shown in Fig. 8, and possibly pointing to a (distant) threshold. From these induction measurements, run $Q$ thus shares more properties with dynamo runs, and thus seems closer to dynamo action than run $P$, while the decay time evolution did not discriminate between the two runs. The analysis of the induction amplitude is thus complementary to the analysis of the relaxation dynamics. The extrapolated threshold for run $Q$ nevertheless remains far out of reach in the experiment. We thus showed that the global magnetic induction response of the system (characterized by $\chi_{2}$ ) can be understood from a (local) analysis of the magnetic induction features close to the impellers. The magnetic behavior in the vicinity of the rotating impellers and the interplay between the sodium flow and the rotating material have a leading role on the global magnetic behavior of the system.

\section{High magnetic permeability versus high electrical conductivity}

In the present subsection, we investigate the differences on dynamo capability between similar runs with parts built from high magnetic permeability (soft iron, $\mu \sim 65$ [30]) and/or high electrical conductivity (copper, 42 times more conducting than stainless steel).

Let us first consider the difference between impellers made entirely of soft iron (runs $R, U, V$ ) and impellers made entirely 


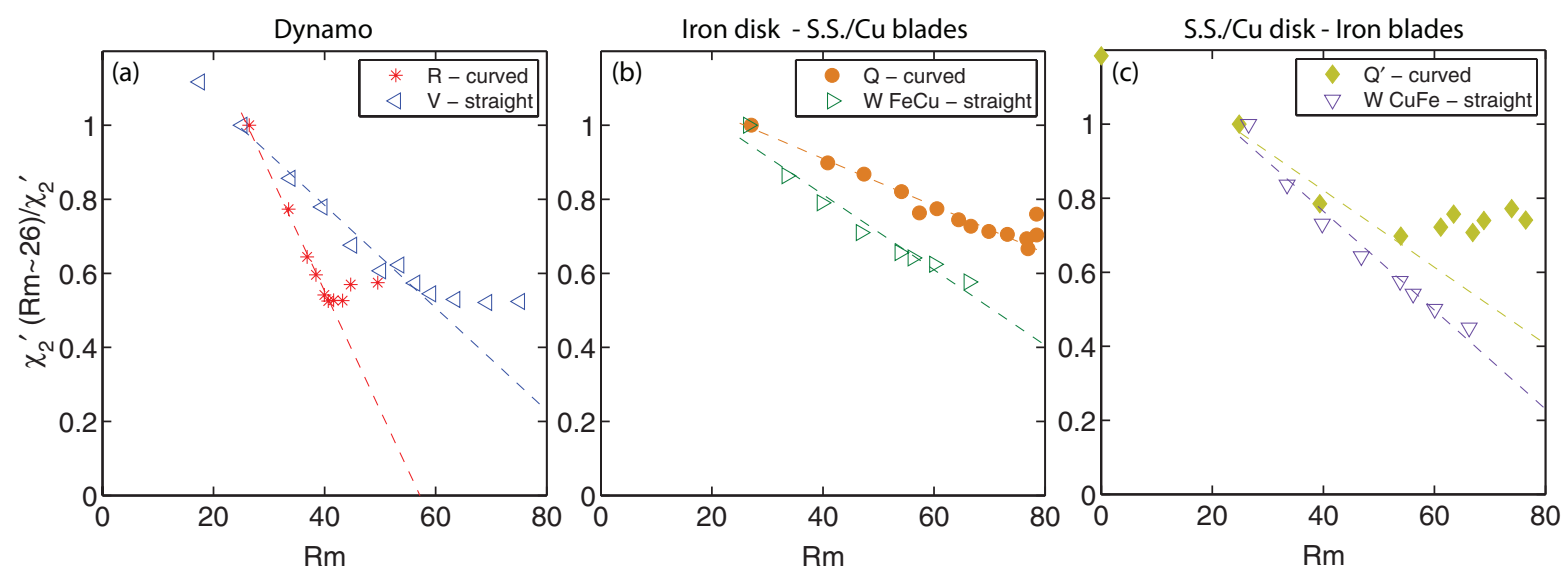

FIG. 10. (Color online) Evolution of $1 / \chi_{2}^{\prime}$ as a function of Rm between different runs; see legend and text for details.

of copper (run $S$ ). As described in Table I, dynamo action has not been observed in run $S$, within the accessible $\mathrm{Rm}$ values $\left(\mathrm{Rm}^{\max } \sim 80\right)$. Unfortunately, due to technical reasons, we were not able to investigate the magnetic relaxation or the magnetic induction. Thus no direct comparison of the magnetic response of the flow driven by high magnetic permeability impellers or by high electrical conductivity impellers is possible.

Let us now consider configurations with mixed impellers, for which the disk and the blades are from different materials (runs $Q, Q^{\prime}, W$ with mixed soft iron/stainless steel and mixed soft iron/copper impellers). This requires a comparison between symmetric configurations runs with curved blades ( $Q$ and $Q^{\prime}$ ) and an asymmetric configuration with straight blades $(W)$.

The first comparison deals with time-decay measurements. Similarly to runs $Q$ and $Q^{\prime}$, a flat evolution of the decay time with $\mathrm{Rm}$ was observed in run $W$ both in exact counterrotation and when only one impeller is rotating, the other one being kept at rest (not shown). In that respect, a higher conductivity of nonmagnetic materials in mixed impellers has no influence on magnetic relaxation.

Though no clue of nearing the dynamo threshold is observed with decay times, it is instructive to investigate in greater details the magnetic induction features. Since configuration $W$ is an asymmetric one, we introduce a slightly modified magnetic susceptibility $\chi_{2}^{\prime}$, for which only one of the shorter probe array is taken into account (i.e., the summation introduced in Sec. III is restricted to probe 1 or probe 4 in Fig. 1). This method has been justified in the previous subsection, where we showed that the global magnetic response of the system is bounded to the magnetic behavior close to the impellers. For symmetric configurations, although the difference between $\chi_{2}$ and $\chi_{2}^{\prime}$ values is around $10 \%$, the linear extrapolation of their evolution with $\mathrm{Rm}$ gave values of the extrapolated threshold within $3 \%$. The investigated runs involve both curved blades configurations (runs $Q, Q^{\prime}$ ) and a straight blade configuration (run $W$ ). We thus first compare the magnetic susceptibility of dynamo runs $R$ (curved blades) and $V$ (straight blades). Figure 10(a) shows the normalized evolution of $1 / \chi_{2}^{\prime}$ for these two dynamo runs: The straight blade configuration is observed to be less efficient than the curved blade configuration to induce magnetic fields (as a consequence run $V$ has a higher threshold than run $R$ ). Figure 10(b) [respectively (c)] shows the normalized evolution of $1 / \chi_{2}^{\prime}$ for the iron disk/stainless steel blades and iron disk/copper blades configurations (respectively stainless steel disk/ iron blades and copper disk/iron blades configurations). For both cases, it is observed that the straight blade configurations are more efficient than the curved blade configurations to induce magnetic fields: The use of high-conductivity materials instead of low-conductivity materials eases electrical current circulation. While the straight blades driven flow is 2.3 less efficient than the curved blades driven flow with full soft-iron impellers [Fig. 10(a)], an increase by a factor 1.6 (respectively 1.3) of the magnetic induction has been observed when stainless steel is replaced by copper for the blades (respectively the disk). An extrapolation of these data lead to an 3.7-fold (respectively 3-fold) increase when stainless steel is replaced by copper for the blades (respectively the disk). In that respect, a higher electrical conductivity of the blades helps in allowing azimuthal currents (excited from helical turbulence or gradient in turbulent properties of the flow close to the impellers) to flow between the blades, thus complementing the benefit of high-permeability rotating disks as proposed in $[15,30]$. However, we emphasize that this effect is not sufficient to reach the dynamo threshold; in addition, no Rm evolution of decay times was observed when the flow is driven by mixed soft iron/copper impellers. The dynamo threshold estimated from the linear extrapolation of $1 / \chi_{2}^{\prime}$ is 120 (respectively 97) for $W \mathrm{FeCu}$ (respectively $W \mathrm{CuFe}$ ); the thresholds estimated for $Q$ and $Q^{\prime}$ are 190 and 125. Magnetic induction is thus more efficient with a rotating nonmagnetic disk fitted with soft-iron blades than with a rotating soft-iron disk fitted with nonmagnetic blades, whatever the conductivity of the nonmagnetic material.

Let us now eventually investigate the effect of a change in the lateral static boundary condition of the cylinder enclosing the flow, as investigated in runs $K$ and $O$. Run $O$ as been described in detail in the last subsection. For run $K$, an evolution of the decay times in the Helmholtz transverse case (applied $m=1$ dipole) with Rm has been observed, however restricted to only two values of $\mathrm{Rm}$ : $\mathrm{Rm}=0$ and $\mathrm{Rm}=51$. A dramatic decrease was observed for $1 / \tau$, from $9 \mathrm{~s}^{-1}$ at $\mathrm{Rm}=0$ to $2.2 \mathrm{~s}^{-1}$ at $\mathrm{Rm}=51$. Within the large uncertainty 
related to the scarce data, a linear extrapolation of this $1 / \tau$ decrease leads to an estimated threshold $\mathrm{Rm}_{\mathrm{c}} \sim 62$.

\section{CONCLUSION}

The dynamo bifurcation is controlled by the magnetic Reynolds number Rm, in a turbulent flow where the mechanical power to be fed to the flow scales as $\mathrm{Rm}^{3}$. For this reason, only restricted $\mathrm{Rm}$ intervals are accessible for any given experimental setup. In these conditions, it is of prime interest to determine the proximity (if any) of a dynamo bifurcation from analysis of induction measurements performed below threshold.

Using a dynamo run in the VKS experiment we have tested here two methods, inspired by techniques applied to critical phenomena and phase transitions. The first one relies on analysis of time-averaged decay times from pulses of applied magnetic field and the observation of critical slowing down close to the dynamo instability threshold. The second one is based on the analysis of the amplitude of magnetic induction, and the existence of a magnetic susceptibility divergence near the dynamo threshold. Despite the high level of broadband turbulent fluctuations, we showed that both methods reveal the proximity of the dynamo bifurcation, and allow for an estimate of its threshold. Decay times and the magnetic induction increase significantly as the magnetic Reynolds number approaches the critical value from below. Similarly to Rayleigh-Bénard convection [8] and previous investigations on the dynamo instability $[17,19,22]$, we verify that one should test the most unstable mode; i.e., the spatial structure of the applied magnetic field must have a significant projection onto the dynamo mode. In VKS, critical slowing down and magnetic susceptibility divergence are both observed for an applied axial dipole or quadrupole, while no effect is observed for an applied field having the geometry of a transverse dipole. We find that both methods yield a similar estimate of the threshold, but about $20 \%$ above the real value. However, these methods are restricted to situations in which the control parameter can be increased to about half the critical value. Moreover, it is well known that the behavior of the magnetic induction can show strong nonlinear effects, as observed in numerical experiment $[35,36]$ (where growth rates of the magnetic energy were observed to evolve nonlinearly with the magnetic Reynolds number), and extrapolation techniques such as the ones presented here may fail. This study nevertheless provides methods that may be confidently applied to currently operated or planned dynamo experiments.

In a second part of the paper, we have used the observations drawn from the dynamo run to the analysis of other flow and boundary conditions tested in the VKS experiment, as reported in Fig. 6. No evidence of a dynamo threshold can be reported from analysis of the response to an axial applied dipolar magnetic field, except for configurations $O, Q, Q^{\prime}$, and $W$ :

(1) Configuration $O$ has the flow surrounded by a stationary ferromagnetic shell. Some increase of the decay time is observed (diamonds in Fig. 7), but this trend is not confirmed in the susceptibility measurement (diamonds in Fig. 8).

(2) Configurations $Q, Q^{\prime}$, and $W$ have rotating ferromagnetic parts in the impellers. In both cases, an increase of the susceptibility is observed (filled circles, Fig. 8), but decay times do not seem to change (filled circles, Fig. 7). In run $W$, it has been observed that changing rotating stainless steel with copper (i.e., higher electrical conductivity) increases magnetic induction, while decay times are not modified.

(3) Run $K$ is a peculiar configuration for which an increase of the decay time has been observed from an applied transverse dipole, though the dynamo threshold was not reached.

These observations confirm the leading role played by boundary conditions in the VKS experiment, particularly the leading effect of rotating ferromagnetic parts.

\section{ACKNOWLEDGMENTS}

We thank the other members of the VKS collaboration, with whom the experimental runs have been performed. We thank M. Moulin, C. Gasquet, A. Skiara, D. Courtiade, J.-F. Point, P. Metz, V. Padilla, and M. Tanase for their technical assistance. We thank the anonymous referee for remarks that raised the discussion of Sec. IV C. This work is supported by ANR 080039-02, Direction des Sciences de la Matière, and Direction de l'Energie Nucléaire of CEA, Ministère de la Recherche, and CNRS. The experiment is operated at CEA/Cadarache DEN/DTN.
[1] F. Charru, Hydrodynamic Instabilities (Cambridge University Press, Cambridge, 2011).

[2] R. P. Behringer and G. Ahlers, Phys. Lett. A 62, 329 (1977).

[3] J. Weisfreid, Y. Pomeau, M. Dubois, C. Normand, and P. Bergé, J. Phys. 39, 725 (1978).

[4] Y. Sawada, Phys. Lett. A 65, 5 (1978).

[5] J. P. Gollub and M. H. Freilich, Phys. Fluids 19, 618 (1976).

[6] R. J. Donnely and K. W. Schwarz, Proc. R. Soc. London A 283, 531 (1965).

[7] G. Pfister and U. Gerdts, Phys. Lett. A 83, 23 (1981).

[8] C. Allain, H. Z. Cummins, and P. Lallemand, J. Phys. Lett. (Paris) 39, L473 (1978).

[9] M. Nishioka, S. Iida, Y. Ichikawa et al., J. Fluid Mech. 72, 731 (1975).

[10] K. Avila, D. Moxey, A. de Lozar, M. Avila, D. Barkley, and B. Hof, Science 333, 192 (2011).
[11] M. L. Dudley and R. W. James, Proc. R. Soc. London A 425, 407 (1989).

[12] F. Ravelet, A. Chiffaudel, F. Daviaud, and J. Leorat, Phys. Fluids 17, 117104 (2005).

[13] C. J. P. Gissinger, Europhys. Lett. 87, 39002 (2009).

[14] A. Giesecke, F. Stefani, and G. Gerbeth, Phys. Rev. Lett. 104, 044503 (2010).

[15] A. Giesecke, C. Nore, F. Stefani, G. Gerbeth, J. Leorat, W. Herreman, F. Luddens, and J.-L. Guermond, New J. Phys. 14, 053005 (2012).

[16] S. Kenjeres and K. Hanjalic, Phys. Rev. Lett. 98, 104501 (2007).

[17] A. K. Gailitis, B. G. Karasev, I. R. Kirillov, O. A. Lielausis, S. M. Luzhanskii, A. P. Ogorodnikov, and G. V. Preslitskii, Magnitnaya Gidrodinamika 23, 349, (1987). 
[18] Yu. B. Ponomarenko, J. Appl. Mech. Tech. Phys. 14, 775 (1973).

[19] A. K. Gailitis, O. Lielausis, S. Dement, E. Platacis, A. Cifersons, G. Gerbeth, T. Gundrum, F. Stefani, M. Christen, H. Hänel, and G. Will, Phys. Rev. Lett. 84, 4365 (2000).

[20] Agris Gailitis, Physics of Plasmas 11, 2838 (2004).

[21] A. Alemany, F. Marty, F. Plunian, and J. Soto, J. Fluid Mech. 403, 263 (2000).

[22] N. L. Peffley, A. Cawthorne, and D. P. Lathrop, Phys. Rev. E 61, 5287 (2001).

[23] R. Monchaux, M. Berhanu, S. Aumaitre, A. Chiffaudel, F. Daviaud, B. Dubrulle, F. Ravelet et al., Phys. Fluids 21, 035108 (2009).

[24] M. Berhanu, G. Verhille, J. Boisson, B. Gallet, C. Gissinger, S. Fauve, N. Mordant et al., Eur. Phys. J. B 77, 459 (2010).

[25] J. Boisson, S. Aumaitre, N. Bonnefoy, M. Bourgoin, F. Daviaud, B. Dubrulle, Ph. Odier, J.-F. Pinton, N. Plihon, and G. Verhille, New J. Phys. 14, 013044 (2012).

[26] B. Gallet, S. Aumaitre, J. Boisson, F. Daviaud, B. Dubrulle, W. Bonnefoy et al., Phys. Rev. Lett. 108, 144501 (2012).
[27] U. Müller, R. Stieglitz, and S. Horanyi, J. Fluid Mech. 498, 31 (2004).

[28] F. Ravelet, M. Berhanu, R. Monchaux, S. Aumaitre, A. Chiffaudel et al., Phys. Rev. Lett. 101, 074502 (2008).

[29] P.-P. Cortet, P. Diribarne, R. Monchaux, A. Chiffaudel, F. Daviaud, and B. Dubrulle, Phys. Fluids 21, 025104 (2009).

[30] G. Verhille, N. Plihon, M. Bourgoin, Ph. Odier, and J.-F. Pinton, New J. Phys. 12, 033006 (2010).

[31] F. Ravelet, B. Dubrulle, F. Daviaud, and P-A. Ratie, Phys. Rev. Lett. 109, 024503 (2012).

[32] P. Frick, V. Noskov, S. Denisov, and R. Stepanov, Phys. Rev. Lett. 105, 184502 (2010).

[33] V. Noskov, S. Denisov, R. Stepanov, and P. Frick, Phys. Rev. E 85, 016303 (2012).

[34] K. Rahbarnia, B. P. Brown, M. M. Clark, E. J. Kaplan, M. D. Nornberg et al., ApJ 759, 80 (2012).

[35] C. Nore, M.-E. Brachet, H. Politano, and A. Pouquet, Phys. Plasmas 4, 1 (1997).

[36] Y. Ponty, P. D. Mininni, J.-F. Pinton, H. Politano, and A. Pouquet, New J. Phys. 9, 296 (2007). 